\title{
An array of gut-on-a-chips for drug development
}

Nureddin Ashammakhi ${ }^{1, *}$, MD, FRCSEd, PhD

Mohammed Elmusrati², Full Professor, DSc (Tech),

${ }^{1}$ Department of Surgery, Oulu University, Oulu, Finland.

${ }^{2}$ School of Technology and Innovations, University of Vaasa, Finland.

$\left(^{*}\right)$ Corresponding author

Nureddin Ashammakhi

Department of Surgery, Oulu University Hospital, P.O. Box 22, FI-90220 Oulu, Finland

Tel. +358 8 3152011, E-mail: nureddin.ashammakhi@oulu.fi

\section{Author contribution}

NA initiated the idea and work. Both NA and ME contributed to intellectual and writing content of the MS.

\section{Disclosure}

No conflict of interest. 


\section{Abstract}

Drug development is a time and cost-consuming long process. Conventionally, this involves the use of two-dimensional (2D) cell culture and animal testing. However, these methods often fail to mimic normal human physiology and drug metabolism, and sometimes complications may occur later because they do not appear in preclinical studies. With recent development of organ-on-a-chip devices, these biomimetic systems try to recapitulate human physiology more than other methods, and promise to largely replace animal studies and reduce costs and complications. Because gut is an important route for drug administration and absorption, gut-on-a-chip platform was developed. To develop a high throughput system, a recent work reported on using a multiplication array of several chip devices, demonstrating the efficiency in studying drug effect on gut barrier function as compared to conventional methods. Huge data was generated, and thus new multidisciplinary approach is needed where data collection, communication and analysis platforms need to be developed. This will enable validation of organ-on-a-chip approach and lay basis for its establishment as efficient and cost-effective tool in drug development. Because of the importance of the work, this commentary highlights important aspects of the topic and try to stimulate new the development of innovations in the field.

Keywords. Drug development, Drug testing, Gut, Microfluidics, Organ-on-a-chip 


\section{Introduction}

Current methods for assessing toxicity rely on the use of 2D culture and on the use of animal experiments. It is a long and costly process to get single medicine to the market. With new EU regulation (REACH policy for Registration, Evaluation, and Authorization of Chemicals) requesting the testing for toxicity of $\sim 30,000$ chemicals, we will need about $2.5-54$ million animals ${ }^{1}$ to use for testing [costing about 1.3-9.3 trillion Euros ${ }^{2,3}$. Animal studies are costly, slow, associated with ethical questions and fail to predict human responses. Using animals is limited also by species-dependent variations. It is also difficult to extrapolate results obtained from animal studies to humans ${ }^{4}$. Unfortunately, many drugs that may proof safe in conventional cell culture studies and in animal studies, result in side effects appearing later during clinical trials or even after their approval and use. Thus, using a more biomimetic testing method such as tissue or organ on chip platform is appealing. In new US regulations, e.g. Toxicology in the 21st Century (Tox21) program calls to move away from animal studies 5 .

\section{Gut-on-a-chip}

With the recent advances made in microfluidics ${ }^{6}$ and their application in developing lab-ona-chip ${ }^{7}$ which was then combined with cells to have "organ-on-a-chip" ${ }^{8}$ devices, it is becoming evident that such devices can be used as an alternative to animal experiments and provide large volume of data and can possibly be more biomimetic that animal experiments themselves as they employ human-derived cells.

Because many drugs are absorbed through the gut, gut-on-a-chip devices developed in $2012-2013^{9,10}$ that has also flora included ${ }^{11}$ to create such a testing platform. The system is 
comprised of intestinal epithelial cells cultured in one side of a membrane in the middle of a microchannel contained in a microchip. Fluid flows on both side to represent luminal and basal sides of the epithelial lining of the gut (Figure $1 a \& b$ ). This achievement represents important an important milestone that following the idea developed by the same group in 2010 for lung-on-a-chip ${ }^{8}$.

In addition to organ-on-a-chip models mimicking normal physiology, organon-a-chip models for pathological conditions such as inflammation ${ }^{12}$, thrombosis ${ }^{13}$, degeneration ${ }^{14}$, and cancer ${ }^{15}$ can also be developed and used for drug development studies.

\section{Multiplied organ-on-a-chip devices}

In next important logical step, which also constitutes the second milestone, recently Trietsch et al. reported on a new device comprising an array of gut-on-a-chip joined together (357 gut tubes in 10 plates) and the efficiency of this setup in testing for drug toxicity was demonstrated ${ }^{16}$.

It is for the first time that such comprehensive method is used for assessing drug concentration and exposure time on the integrity of intestinal epithelial barrier. It is the largest ever reported organ-on-a-chip system that used more than 20,000 data-points. The study opens new avenues for efficient and reliable future drug testing and development. Trietsch et al. ${ }^{16}$ have cultured human intestinal colorectal adenocarcinoma cell line (Caco-2) in one microfluidic channel and were separated by collagen-type-l gel from fluid flow channel (Figure 1c-r). Ten plates, each comprising 40 leak-tight tubes in parallel, were used. Cells exhibited differentiation characteristics and markers such as brush border, tight 
junctions, fluid transport and intact epithelial barrier function. Cell polarization against the gel was also observed. Fluorescent probes were used to assess barrier integrity and its druginduced loss of integrity with resulting leak at day 4 of culture. Barrier integrity loss was assessed by exposing the apical side of cell lining to Staurosporine (inducer of apoptosis) and aspirin (affects tight junctions) for $125 \mathrm{~h}$. Comparison experiments were performed in conventional Transwell and showed lower sensitivity to detect barrier disruption as compared to organ-on-a-chip setup. A clear drug concentration-response effect was also detected. Although such organ-on-a-chip devices offer a new platform for drug testing, the technology needs validation against current standards and clinical results to establish a solid recognized method of testing in drug development. This work ${ }^{16}$ is thus also important contribution in this direction.

\section{Data Analysis}

While the current work multiplies the organ-on-a-chip units many times creating huge and unprecedented amount of data, it requires parallel development of appropriate data analysis and management system as well as modelling. New multidisciplinary approach is thus needed and should be based on developing at least the following layers: 1 . Sensing technologies, to sense and measure the cell responses accurately; 2 . Communication technology, to collect time series data from maybe tens of thousands of sensors effectively; 3. Big data analysis and mining using effective artificial intelligence and machine learning algorithms; 4. Biochemical analysis and modelling, for drug design, testing and evaluation; And, 5. Pathological models for the assessment of changes and effect of drug(s) in pathological situation(s). There is a different optimisation and working procedures of each layer. However, cross-layer optimisation would be crucial to enhance the efficiency and the 
speed of the whole process. Another possible important application of the system is the online medical drug design or fine-tuning. The testing phase will just give us a conclusion about the validity of the drug and its effectiveness as well as possible side effects on the short and long-terms. However, with appropriately large number of data-points with many related sensors involved and online data analysis (using for example advanced machine learning and intelligent automation), it could be possible to modify the drug (e.g., chemical structure, compound concentration, design process) online. Within the closed-loop intelligent operations of drug-modifications and their impacts on cells, we will be able to perform thousands of directed (optimised) trials within proper duration of time. Current technical systems in terms of reliable electronics, sensors accuracy, computing facilities, smart algorithms, and intelligent micro-automated systems can be integrated as the foundation of the next generation drug design and development.

\section{Conclusions}

Gut on chip was developed to assess drug testing and aid drug development. To achieve high throughput, multiplications array of these devices were recently developed and its efficiency in such process was demonstrated. Data generated is huge and needs development of proper management. Multidisciplinary approach is necessary to develop novel functional platforms for drug testing and development based in multiplied organ-onchips, data collection, communication and analysis. Costs will hence, be saved, efficient medicines developed and risks to patients tremendously reduced.

1. Hartung T, Rovida C. Chemical regulators have overreached: The costs--both in animal lives and euros--of the european REACH legislation on chemical testing are escalating. 
thomas hartung and costanza rovida argue for a suspension of certain toxicity tests. Nature. 2009;460(7259):1080.

2. Blaauboer B, Andersen M. The need for a new toxicity testing and risk analysis paradigm to implement REACH or any other large scale testing initiative. Arch Toxicol. 2007;81(5):385-387. http://www.ncbi.nlm.nih.gov/pubmed/17262219. doi: 10.1007/s00204006-0175-0.

3. Greim H, Arand M, Autrup H, et al. Toxicological comments to the discussion about REACH. Arch Toxicol. 2006;80(3):121-124.

http://www.ncbi.nlm.nih.gov/pubmed/16411136. doi: 10.1007/s00204-005-0039-z.

4. Hartung T. Toxicology for the twenty-first century. Nature. 2009;460(7252):208-212. http://dx.doi.org/10.1038/460208a. doi: 10.1038/460208a.

5. Melanie Adler, Susanne Ramm, Marc Hafner, et al. A quantitative approach to screen for nephrotoxic compounds in vitro. Journal of the American Society of Nephrology : JASN. 2016;27(4):1015-1028. http://www.ncbi.nlm.nih.gov/pubmed/26260164. doi: 10.1681/ASN.2015010060.

6. Whitesides GM. The origins and the future of microfluidics. Nature. 2006;442(7101):368373. http://dx.doi.org/10.1038/nature05058. doi: 10.1038/nature05058.

7. H A Stone, A D Stroock, A Ajdari. Engineering flows in small devices : Microfluidic toward a lab-on-a-chip. Annual Review of Fluid Mechanics. 2004;36:381. https://search.proquest.com/docview/220814839.

8. Dongeun Huh, Benjamin D. Matthews, Akiko Mammoto, Martín Montoya-Zavala, Hong Yuan Hsin, Donald E. Ingber. Reconstituting organ-level lung functions on a chip. Science. 2010;328(5986):1662-1668. http://www.sciencemag.org/content/328/5986/1662.abstract. doi: 10.1126/science. 1188302 . 
9. Kim SH, Lee JW, Choi I, Kim Y, Lee JB, Sung JH. A microfluidic device with 3-d hydrogel villi scaffold to simulate intestinal absorption. Journal of nanoscience and nanotechnology. 2013;13(11):7220. http://www.ncbi.nlm.nih.gov/pubmed/24245233.

10. Kim HJ, Ingber DE. Gut-on-a-chip microenvironment induces human intestinal cells to undergo villus differentiation. Integrative biology : quantitative biosciences from nano to macro. 2013;5(9):1130. http://www.ncbi.nlm.nih.gov/pubmed/23817533. doi:

$10.1039 / \mathrm{c} 3 \mathrm{ib} 40126 \mathrm{j}$.

11. Hyun Jung Kim, Dongeun Huh, Geraldine Hamilton, Donald E Ingber. Human gut-on-achip inhabited by microbial flora that experiences intestinal peristalsis-like motions and flow. Lab on a chip. 2012;12(12):2165-2174. http://www.ncbi.nlm.nih.gov/pubmed/22434367. doi: $10.1039 / \mathrm{c} 2 \mathrm{lc} 40074 \mathrm{j}$.

12. Ribas J, Zhang YS, Pitrez PR, et al. Biomechanical strain exacerbates inflammation on a Progeria- on- a- Chip model. Small. 2017;13(15):n/a.

http://onlinelibrary.wiley.com/doi/10.1002/smll.201603737/abstract. doi: 10.1002/smll.201603737.

13. Zhang YS, Davoudi F, Walch P, et al. Bioprinted thrombosis-on-a-chip. Lab Chip. 2016;16(21):4097-4105. doi: 10.1039/C6LC00380J.

14. Kilinc D, Peyrin J, Soubeyre V, et al. Wallerian-like degeneration of central neurons after synchronized and geometrically registered mass axotomy in a three-compartmental microfluidic chip. Neurotox Res. 2011;19(1):149-161.

http://www.ncbi.nlm.nih.gov/pubmed/20162389. doi: 10.1007/s12640-010-9152-8.

15. Bersini S, Jeon JS, Dubini G, et al. A microfluidic 3D in vitro model for specificity of breast cancer metastasis to bone. Biomaterials. 2014;35(8):2454.

http://www.ncbi.nlm.nih.gov/pubmed/24388382. doi: 10.1016/j.biomaterials.2013.11.050. 
16. Sebastiaan J Trietsch, Elena Naumovska, Dorota Kurek, et al. Membrane-free culture and real-time barrier integrity assessment of perfused intestinal epithelium tubes. Nature Communications. 2017;8:1. https://search.proquest.com/docview/1929060080. doi: 10.1038/s41467-017-00259-3.

\section{Figure legends}

Figure 1.

a) Schematic illustration of the gut-on-a-chip device which comprises several channels. The central channel has a flexible porous ECM-coated membrane. Gut epithelial cells were cultured on the top of the membrane. Vacuum chambers are seen laying on both sides of the central channel. Reproduced with kind permission form Kim et al. ${ }^{11}$, b) Schematic illustration showing the formation of intestinal villus structure. Reproduced with kind permission form Kim et al. ${ }^{10}$

c) OrganoPlate system having 40 microfluidic channel networks. The inlay shows top view of the 384-well plate format device. d) Single microfluidic network having three channels joined in the center. e, $g, i, k$ ) Horizontal projection and $f, h, j, I$ vertical cross section of central region for the following steps. e, f) An extracellular matrix (ECM) gel (light gray) is patterned by two phaseguides (dark gray). g, h) Lanes adjacent to the ECM gel had culture medium introduced into them. One of the lanes has cells. i, j) Cells were allowed to settle against the ECM gel by placing the plate on its side. k, l) Following flow, cells form a confluent layer that lined the surface of channel and gel. m) Schematic of the chip center comprising a tubule, ECM gel and a perfusion lane. Two phaseguides (white bars) define the three distinct lanes in the central channel. At its apical side, the tubule has a perfusable 
bioRxiv preprint doi: https://doi.org/10.1101/273847- this version posted February 28,2018 . The copyright holder for this preprint (which was not certified by peer review) is the author/funder, who has granted bioRxiv a license to display the preprint in perpetuity. It is made available under aCC-BY-NC-ND 4.0 International license.

lumen. n-r) Images (phase-contrast) of the tubular structure formation at day 0, 1, 4, 7, and

11, respectively. Scale bars: $100 \mu \mathrm{m}$. Reproduced with kind permission form Trietsch et al.

(1).

a
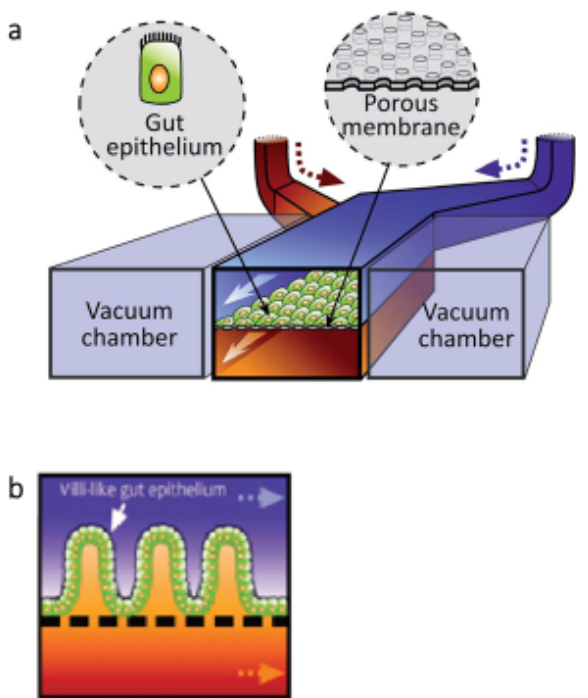

c
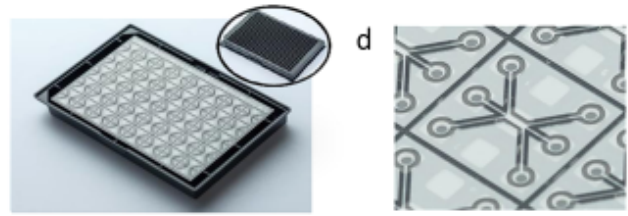

$\mathrm{e}$

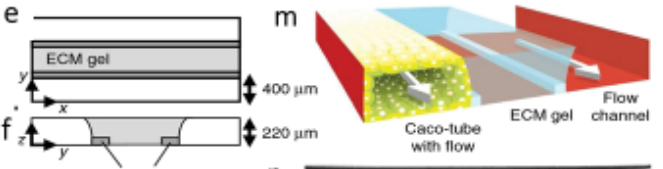

$\mathrm{g}$
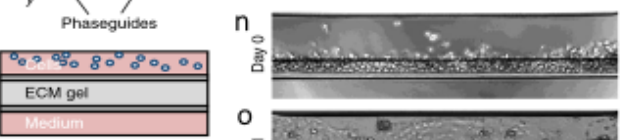

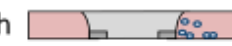
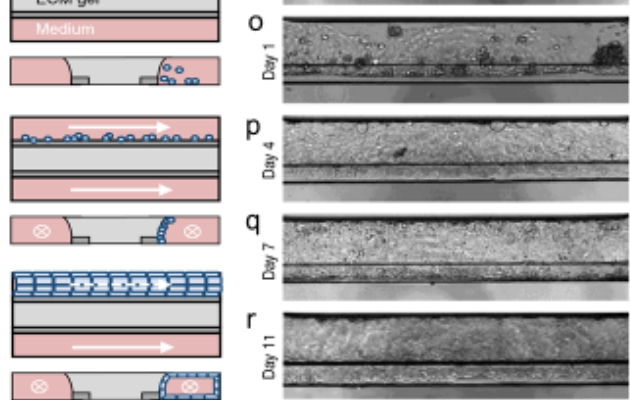